\title{
The Combination of the Fetal Fibronectin Bedside Test and Cervical Length in Preterm Labor Is Useful for Prediction of Preterm Birth
}

\author{
Monya Todesco $^{1 *}$, Moritz Hartog2\#, Thomas Fabbro ${ }^{3}$, Olav Lapaire ${ }^{4}$, Irene M. Hoesli ${ }^{4}$ \\ ${ }^{1}$ Department of Obstetrics and Gynecology, Kantonsspital Aarau, Aarau, Switzerland \\ ${ }^{2}$ Department of Obstetrics and Gynecology, Marien Hospital Witten, Witten, Germany \\ ${ }^{3}$ Clinical Trial Unit, University of Basel, Basel, Switzerland \\ ${ }^{4}$ Department of Obstetrics, University of Basel, Basel, Switzerland \\ Email: *monya.todesco@ksa.ch,moritz_hartog@gmx.de,Olav.Lapaire@usb.ch, Irene.Hoesli@usb.ch, \\ Thomas.Fabbro@usb.ch
}

Received 31 August 2015; accepted 8 November 2015; published 11 November 2015

Copyright (C) 2015 by authors and Scientific Research Publishing Inc.

This work is licensed under the Creative Commons Attribution International License (CC BY).

http://creativecommons.org/licenses/by/4.0/

(c) (i) Open Access

\begin{abstract}
Objective: To determine the value of fetal fibronectin (FFN), cervical length (CL) measurement and their combination as binary predictors for preterm birth (PB) in women with preterm labour (PTL) between $24+0$ and $34+0$ weeks. Methods: One hundred fifty-nine patients with signs of PTL (singleton pregnancies $(\mathrm{SP})=125$, twin pregnancies $(\mathrm{TP})=34$ ) were evaluated in a retrospective study. Inclusion criteria were contractions $>4 / 20 \mathrm{~min}$, intact membranes, no bleeding. The cut-off was $\geq 50 \mathrm{ng} / \mathrm{ml}$ for FFN and $\leq \mathbf{2 0} \mathbf{~ m m}$ for CL measured by transvaginal ultrasound. The primary outcome variable was delivery within 7 days from admission. Results: We evaluated 125 SPs and 34 TPs. In SPs, both methods had a sensitivity of $80 \%$; the specificity was $82 \%$ for FFN, and $50 \%$ for CL. For the combination of both tests sensitivity was $80 \%$ and specificity $88 \%$. In TPs, the sensitivity of both tests was lower (FFN 33\%, CL 67\%) but the combination of both tests represented the highest result for specificity (77\% compared to $68 \%$ for FFN alone and $32 \%$ for CL alone). Conclusion: The combination of FFN and CL in PTL results in a significant higher specificity in SPs. In TPs the performance of the tests is less accurate.
\end{abstract}

\section{Keywords}

Preterm Labor, Fetal Fibronectin, Cervical Length, Preterm Birth

\footnotetext{
${ }^{*}$ Corresponding author.

"First and second authors contributed equally to the study.
}

How to cite this paper: Todesco, M., Hartog, M., Fabbro, T., Lapaire, O. and Hoesli, I.M. (2015) The Combination of the Fetal Fibronectin Bedside Test and Cervical Length in Preterm Labor Is Useful for Prediction of Preterm Birth. Open Journal of Obstetrics and Gynecology, 5, 746-753. http://dx.doi.org/10.4236/ojog.2015.513105 


\section{Introduction}

Preterm birth (PTB) is the leading cause of neonatal mortality and morbidity. Therefore, women presenting with signs of preterm labour (PTL) but intact membranes are often hospitalised and receive tocolytics and corticosteroids for lung maturation. The clinical diagnosis of PTL that precedes PTB has been characterised by its inaccuracy, with about two thirds of women presenting with PTL ultimately delivering at term. Correct identification of a mother at risk for a PTB allows risk specific treatment, stabilisation, and referral to a perinatal centre for achieving optimal neonatal outcome. It avoids otherwise unnecessary transport of the patient, potential harmful interventions and costly hospitalisations.

Several predictive markers have been proposed detecting women at risk for PTB. The biophysical sonographic measurement of cervical length (CL) and the biochemical assays for fetal fibronectin (FFN) appear to be the most promising and have been assessed in multiple studies [1] [2].

FFN is a normal constituent of the extracellular matrix of the maternal-fetal interface. This high molecular weight glycoprotein seems to play an essential role in maintaining the integrity of the chorionic-decidual interface. It is accepted that the disruption of the maternal-fetal interface often precedes the onset of PTL and results in the release of FFN into the cervicovaginal fluid [2] [3].

CL measurement is well established as a predictor for PTB at 20 weeks of gestation in asymptomatic women at increased risk [4] but less evaluated in symptomatic women with PTL using mostly thresholds below $15 \mathrm{~mm}$ or above $30 \mathrm{~mm}$ cervical length [5]. Short cervix reportedly predicts PTD with a sensitivity of $69 \%$ - 83\%, a specificity of $69 \%$ - $88 \%$, and a relatively low positive predictive value (PPV) of $22 \%$ - 54\% in symptomatic women.

The usefulness of both tests has been shown by improving the accuracy of diagnosis and in reducing unnecessary maternal interventions and transferral of patients to a tertiary center. Furthermore, they are easily to apply after minimal training, safe, well accepted by pregnant women and cost effective [6]-[9].

There are, however, conflicting results from retrospective and observational studies, as well as from metaanalysis. Two Cochrane reviews have found no significant association between knowledge of CL results and a lower incidence of PTB, but for FFN an association between knowledge of FFN results and a lower incidence of PTB before 37 weeks was mentioned [10] [11]. Some authors found the combination not superior to either test alone, especially compared to CL measurement [3] [12] [13] and a recent meta-analysis found the cervicovaginal FFN assay to have limited accuracy in predicting PTB within 7 days of sampling in symptomatic pregnant women [14].

The discordant results of these studies illustrate the predicament for the clinical routine situation. Furthermore, the studies use different cut-off values and different ranges of examined weeks of gestation, which makes them even more difficult to compare and interpret.

Our objective was to determine whether the combination of the two tests was superior to each test alone as a prediction of preterm birth within seven days in symptomatic women with singleton and twin pregnancies.

\section{Methods}

This was a retrospective study with reviewed case notes conducted at two Swiss tertiary centres (center A 2000 deliveries/year and center B 1300 deliveries/year) from 2005-2008. Included were patients with PTL (at least 4 contractions within 20 minutes), between $24+0$ and $33+6$ weeks of gestation with a twin or singleton pregnancy and who had a documented assessment of FFN and CL. Gestational age was confirmed by first trimester ultrasound. Exclusion criteria were preterm premature rupture of membranes, cervical dilation $>3 \mathrm{~cm}$, cerclage, vaginal bleeding, vaginal examination or intercourse within $24 \mathrm{~h}$ before FFN measurement, intrauterine fetal demise, fetal malformation, intrauterine growth restriction $<5$ th percentile, preeclampsia, and uterine malformation. At presentation to the hospital FFN was collected during speculum examination from the posterior fornix and the qualitative detection of FFN (Quikcheck FFN, Hologic, Marlborough, MA, USA) was performed as a rapid qualitative bedside test, described by the manufacturer (http://www.ffntest.com/hcp/testing/quikcheck.html). Subsequently transvaginal ultrasound (using a $4-8 \mathrm{MHz}$ vaginal probe, Logiq or Voluson, GE healthcare or Alpha 5, Aloka) was carried out by the attending physician and CL was measured with an empty bladder as described in earlier publications and the shortest functional CL was used [15].

The cut off values used were FFN $\geq 50 \mathrm{ng} / \mathrm{ml}$ and $\mathrm{CL} \leq 20 \mathrm{~mm}$ on transvaginal ultrasound. The CL cut-off value of $20 \mathrm{~mm}$ was chosen, since it is commonly used in both singleton pregnancies (SP) and twin pregnancies 
(TP).

Results of the FFN tests were available to the attending clinicians who were free to use this information at their discretion for clinical management. However only CL was part of the local clinical management protocols, which physicians followed also with regard to the administration of tocolysis and corticosteroids for fetal lung maturation.

The primary outcome was the occurrence of PTB within 7 days ( $\leq 7$ days) from admission. The two tests were evaluated separately as well as in combination, considering the combined test positive if both tests were positive (“AND” combination). As the management of preterm labor was not changed during the study period it was described as part of the assessment of the institutional quality of the service and therefore ethical approval was not obtained.

Patients with SP and with TP were analysed separately. Sensitivity, specificity and likelihood ratios, as well as positive and negative predictive values were computed, together with $95 \%$ confidence intervals (CI) according to Blaker 2000 and were tested with the McNemar-Test at a significance level of 0.05 . The data were analysed using the statistical software R.

\section{Results}

A total of 159 patients were included, of which 125 were SPs and 34 TPs. They presented with PTL at a median age of gestation of $29+5$ weeks and $29+1$ weeks in SP and TP, respectively. Demographic characteristics of the women, which were comparable between singleton and twin pregnancies and the main pregnancy outcomes, are displayed in Table 1.

Table 1. Baseline characteristics.

\begin{tabular}{|c|c|c|c|c|}
\hline & & $\mathrm{N}$ & Singleton $N=125$ & Twins $\mathrm{N}=34$ \\
\hline Age & years & 159 & 253033 & 303335 \\
\hline BMI & $\mathrm{kg} / \mathrm{m}^{2}$ & 159 & 232629 & 242729 \\
\hline History of preterm birth & & 159 & $11.2 \%(14)$ & $5.9 \%(2)$ \\
\hline Prity & & 159 & & \\
\hline 0 & & & $52.0 \%(65)$ & $64.7 \%(22)$ \\
\hline 1 & & & $28.8 \%(36)$ & $17.6 \%(6)$ \\
\hline$>1$ & & & $19.2 \%(24)$ & $17.6 \%(6)$ \\
\hline Gravidity & & 159 & & \\
\hline 1 & & & $48.0 \%(60)$ & $61.8 \%(21)$ \\
\hline 2 & & & $20.0 \%(25)$ & $14.7 \%(5)$ \\
\hline$>2$ & & & $32.0 \%(40)$ & $23.5 \%(8)$ \\
\hline Gestation age at hospitalization & day & 159 & $28+129+531+6$ & $27+029+130+4$ \\
\hline Cervical length & $\mathrm{mm}$ & 159 & 14.020 .030 .0 & 13.018 .022 .8 \\
\hline Lung maturation & & 159 & $65.6 \%(82)$ & $91.2 \%(31)$ \\
\hline Gestation age at birth & day & 159 & $36+338+139+3$ & $33+135+136+2$ \\
\hline Fetal weight at birth & g & 157 & 277030503400 & 196222022551 \\
\hline Days of hospitalization & day & 132 & 2.004 .008 .00 & 4.757 .5013 .25 \\
\hline Preterm birth before $37+0$ weeks & & 159 & $31.2 \%(39)$ & $85.3 \%(29)$ \\
\hline Preterm birth before $34+0$ weeks & & 159 & $8.8 \%(11)$ & $32.4 \%(11)$ \\
\hline Tocolysis & & 159 & $84.8 \%(106)$ & $91.2 \%(31)$ \\
\hline Vaginal bacterial or yeast colonization & & 159 & $37.6 \%(47)$ & $26.5 \%(9)$ \\
\hline
\end{tabular}

a b c represent the lower quartile a, the median b, and the upper quartile c for continuous variables. $\mathrm{N}$ is the number of non-missing values. Numbers after percents are frequencies. 
PTB within 7 days occurred in 5/125 of SP and in 3/34 of TP. In SP and TP CL $\leq 20$ mm occurred in 64/125 and 23/34, respectively. FFN was positive in 26/125 of SP and in 11/34 of TP. Tocolytics were administered in 106/125 (84.8\%) of SP and in 31/34 (91.2\%) of TP in PTL. The PTB rate < 37 weeks in women with symptoms of labor was $31.2 \%$ for SP and $85.3 \%$ for TP. Table 2 and Table 3 present the baseline characteristics according to the results of FFN and cervical length measurement.

In SP both methods had a sensitivity of $80 \%$ with 95\%-CI: [34.3\%; 99\%] for the given time period of 7 days. The specificity of the CL test alone was 50\% with 95\%-CI: [40.8\%; 59.2\%] which was significantly smaller than the specificity of the combination of CL and FFN-test of 88.3\% with 95\%-CI: [81.5; 93.2] (McNemar-Test, $\mathrm{p}<0.001)$. Also, the specificity of the FFN test alone (81.7\% [73.9\%; 87.8\%]) was significantly smaller than the specificity of the combination of cervix and FFN-test (McNemar-Test, $\mathrm{p}=0.013$ ).

The Positive Predictive Value for CL was 6.2\% [2.2\%; 15.1\%], for FFN 15.4 [5.4; 34.1] and for the combination 22.2 [8\%; 47.1\%].

The Negative Predictive Value for CL was 98.4\% [91.6\%; 99.9\%] and for FFN 99.0\% [94.8\%; 99.9\%], for the combination 99.1\% [95.2\%; 99.9\%]. In Figure 1, the estimates are shown together with the corresponding 95\% confidence interval.

In TP, the sensitivity of both tests was less (FFN 33\% and CL 67\%) compared to SPs, but again the combination of the two tests showed the highest result for specificity (77\% compared to $68 \%$ for FFN alone and 32\% for CL alone).

Table 2. Baseline characteristics separated by the test out comes for singletons.

\begin{tabular}{|c|c|c|c|c|c|c|}
\hline & & $\mathrm{N}$ & $\begin{array}{c}\mathrm{fFN}+, \mathrm{CL}+ \\
\mathrm{N}=18\end{array}$ & $\begin{array}{c}\mathrm{fFN}+, \mathrm{CL}_{-}^{-} \\
\mathrm{N}=8\end{array}$ & $\begin{array}{c}\mathrm{fFN}-, \mathrm{CL}+ \\
\mathrm{N}=46\end{array}$ & $\begin{array}{c}\mathrm{fFN}-, \mathrm{CL}^{-} \\
\mathrm{N}=53\end{array}$ \\
\hline Age & years & 125 & 293234 & 253033 & 242935 & 253032 \\
\hline BMI & $\mathrm{kg} / \mathrm{m}^{2}$ & 125 & 252831 & 242628 & 232530 & 242528 \\
\hline History of preterm birth & & 125 & $5.6 \%(1)$ & $50.0 \%(4)$ & $8.7 \%(4)$ & $9.4 \%(5)$ \\
\hline Parity & & 125 & & & & \\
\hline 0 & & & $61 \%(11)$ & $50 \%(4)$ & $48 \%(22)$ & $53 \%(28)$ \\
\hline 1 & & & $22 \%(4)$ & $12 \%(1)$ & $35 \%(16)$ & $28 \%(15)$ \\
\hline$>1$ & & & $17 \%(3)$ & $38 \%(3)$ & $17 \%(8)$ & $19 \%(10)$ \\
\hline Gravidity & & 125 & & & & \\
\hline 1 & & & $61 \%(11)$ & $50 \%(4)$ & $44 \%(20)$ & $47 \%(25)$ \\
\hline 2 & & & $17 \%(3)$ & $12 \%(1)$ & $17 \%(8)$ & $24 \%(13)$ \\
\hline$>2$ & & & $22 \%(4)$ & $38 \%(3)$ & $39 \%(18)$ & $28 \%(15)$ \\
\hline Gestation age at hospitalization & day & 125 & $29+130+332+0$ & $28+028+229+3$ & $28+029+031+4$ & $27+530+232+2$ \\
\hline Cervical length & $\mathrm{mm}$ & 125 & 7.812 .016 .0 & 27.031 .534 .8 & 11.214 .017 .0 & 25.030 .034 .0 \\
\hline Lung maturation & & 125 & $94.4 \%(17)$ & $87.5 \%(7)$ & $84.8 \%(39)$ & $35.8 \%(19)$ \\
\hline Gestation age at birth & day & 125 & $34+035+337+5$ & $35+437+138+2$ & $36+638+239+4$ & $37+338+440+0$ \\
\hline Fetal weight at birth & g & 125 & 219528402998 & 256927352995 & 286931703400 & 287031303500 \\
\hline Days of hospitalization & day & 108 & 0.000 .000 .75 & 0.000 .004 .00 & 5.008 .0020 .25 & 1.003 .004 .00 \\
\hline Preterm birth before $37+0$ weeks & & 125 & $67 \%(12)$ & $50 \%(4)$ & $26 \%(12)$ & $21 \%(11)$ \\
\hline Preterm birth before $34+0$ weeks & & 125 & $27.8 \%(5)$ & $12.5 \%(1)$ & $8.7 \%(4)$ & $1.9 \%(1)$ \\
\hline Tocolysis & & 125 & $100.0 \%(18)$ & $87.5 \%(7)$ & $93.5 \%(43)$ & $71.7 \%(38)$ \\
\hline Vaginal bacterial or yeast colonization & & 125 & $44 \%(8)$ & $25 \%(2)$ & $30 \%(14)$ & $43 \%(23)$ \\
\hline
\end{tabular}

a b c represent the lower quartile a, the median b, and the upper quartile c for continuous variables. $\mathrm{N}$ is the number of non-missing values. Numbers after percents are frequencies. 
Table 3. Baseline characteristics separated by the test out comes for twins.

\begin{tabular}{|c|c|c|c|c|c|c|}
\hline & & $\mathrm{N}$ & $\begin{array}{c}\mathrm{fFN}+, \mathrm{CL}+ \\
\mathrm{N}=7\end{array}$ & $\begin{array}{c}\mathrm{fFN}+, \mathrm{CL}- \\
\mathrm{N}=4\end{array}$ & $\begin{array}{c}\mathrm{fFN}-, \mathrm{CL}+ \\
\mathrm{N}=16\end{array}$ & $\begin{array}{c}\mathrm{fFN}-, \mathrm{CL}- \\
\mathrm{N}=7\end{array}$ \\
\hline Age & years & 34 & 323437 & 283236 & 303434 & 303334 \\
\hline BMI & $\mathrm{kg} / \mathrm{m}^{2}$ & 34 & 242431 & 272728 & 242630 & 232728 \\
\hline History of preterm birth & & 34 & $14 \%(1)$ & $25 \%(1)$ & $0 \%(0)$ & $0 \%(0)$ \\
\hline Parity & & 34 & & & & \\
\hline 0 & & & $71.4 \%(5)$ & $50.0 \%(2)$ & $68.8 \%(11)$ & $57.1 \%(4)$ \\
\hline 1 & & & $0.0 \%(0)$ & $25.0 \%(1)$ & $25.0 \%(4)$ & $14.3 \%(1)$ \\
\hline$>1$ & & & $28.6 \%(2)$ & $25.0 \%(1)$ & $6.2 \%(1)$ & $28.6 \%(2)$ \\
\hline Gravidity & & 34 & & & & \\
\hline 1 & & & $71 \%(5)$ & $50 \%(2)$ & $69 \%(11)$ & $43 \%(3)$ \\
\hline 2 & & & $0 \%(0)$ & $25 \%(1)$ & $12 \%(2)$ & $29 \%(2)$ \\
\hline$>2$ & & & $29 \%(2)$ & $25 \%(1)$ & $19 \%(3)$ & $29 \%(2)$ \\
\hline Gestation age at hospitalization & day & 34 & $27+129+130+1$ & $30+231+131+6$ & $26+028+430+2$ & $27+028+629+1$ \\
\hline Cervical length & $\mathrm{mm}$ & 34 & 101314 & 252526 & 121619 & 223033 \\
\hline Lung maturation & & 34 & $100 \%(7)$ & $100 \%(4)$ & $100 \%(16)$ & $57 \%(4)$ \\
\hline Gestation age at birth & day & 34 & $31+635+336+2$ & $33+034+035+3$ & $34+235+336+2$ & $32+435+035+5$ \\
\hline Fetal weight at birth & g & 32 & 173823652844 & 213322302506 & 207121942551 & 187420312406 \\
\hline Days of hospitalization & day & 24 & 9.09 .09 .0 & & 4.87 .015 .2 & 4.08 .08 .0 \\
\hline Preterm birth before $37+0$ weeks & & 34 & $71 \%(5)$ & $100 \%(4)$ & $88 \%(14)$ & $86 \%(6)$ \\
\hline Preterm birth before $34+0$ weeks & & 34 & $43 \%(3)$ & $50 \%(2)$ & $19 \%(3)$ & $43 \%(3)$ \\
\hline Tocolysis & & 34 & $100.0 \%(7)$ & $100.0 \%(4)$ & $93.8 \%(15)$ & $71.4 \%(5)$ \\
\hline Vaginal bacterial or yeast colonization & & 34 & $43 \%(3)$ & $50 \%(2)$ & $12 \%(2)$ & $29 \%(2)$ \\
\hline
\end{tabular}

a b c represent the lower quartile a, the median $\mathrm{b}$, and the upper quartile $\mathrm{c}$ for continuous variables. $\mathrm{N}$ is the number of non-missing values. Numbers after percents are frequencies.

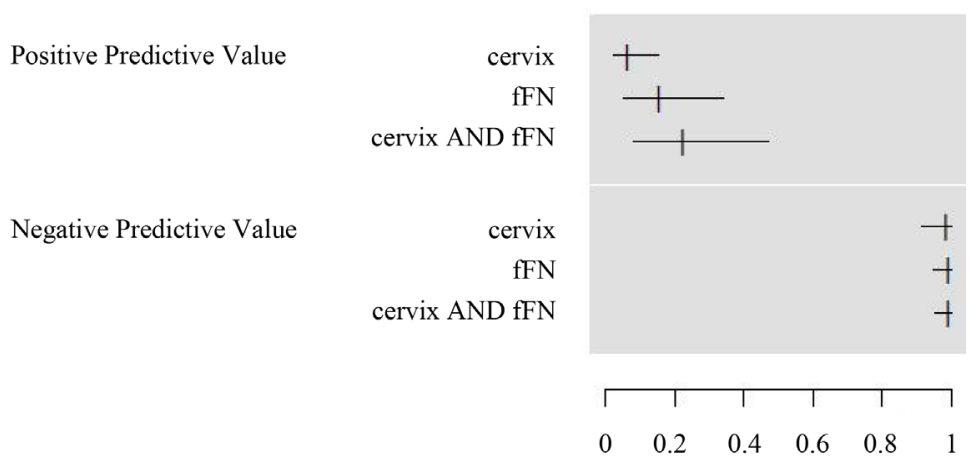

Figure 1. Singleton pregnancies, positive and negative predictive values.

The Positive Predictive Value for CL was 8.7\% [1.60\%; 27.4\%], for FFN 9.1\% [0.50\%; 40.1\%] and for the combination 0\% [0\%; 37.7\%]. The Negative Predictive Value for CL was 90.9\% [59.9\%; 99.5\%], for FFN 91.3\% [72.6\%; 98.4\%] and for combination 88.9\% [71.1\%; 96.9\%] (Figure 2).

The NPV was similar for both tests alone and the combination of the two.

Likelihood ratios are demonstrated in Figure 3 and Figure 4 (in all figures the estimates are shown together with the corresponding 95\% confidence interval). 
Positive Predictive Value

Negative Predictive Value

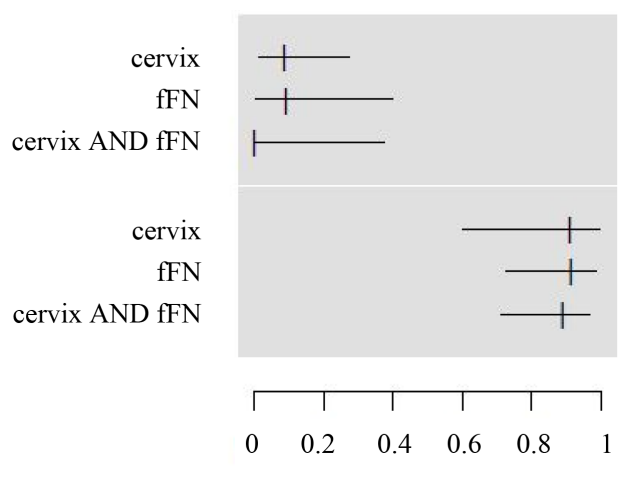

cervix

Figure 2. Twin pregnancies, positive and negative predictive values.

Positive Likelihood Ratio

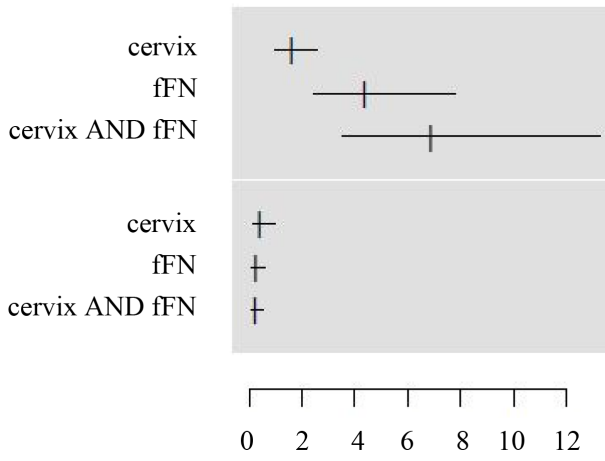

Figure 3. Singleton pregnancies, positive and negative likelihood ratios.

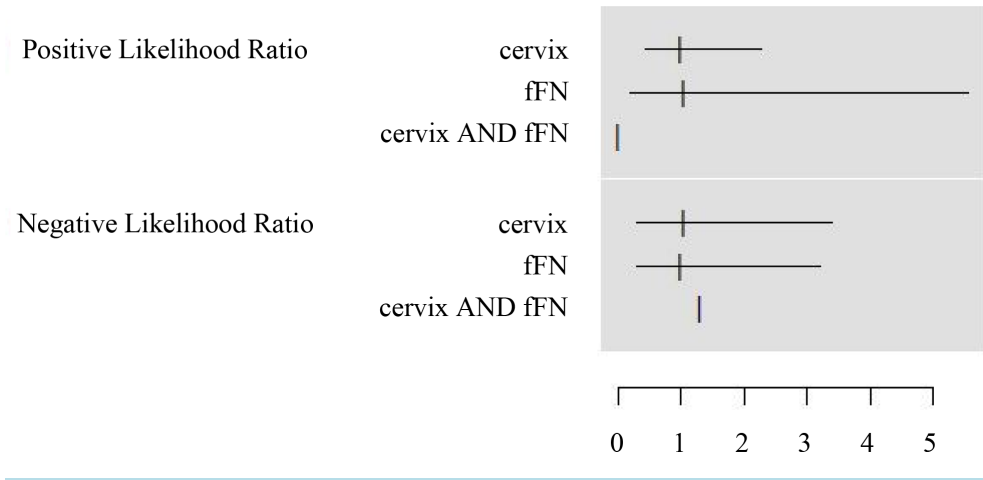

Figure 4. Twin pregnancies, positive and negative likelihood ratios.

For the combined test women with a positive result received tocolytics in $100 \%$ compared to those with two negative tests $(7.41 \%)$. Not only the rate for preterm birth within seven days was higher in this group but also the rate for PTB $<34$ and $<37$ weeks.

\section{Discussion}

The findings of this study clearly demonstrate the advantage of the combined test of a positive FFN and a short CL for the identification of patients with true preterm labour. A very small part of women with SP and clinical symptoms of labor delivered within 7 days. Compared to the clinical assessment we had a modest but higher positive predictive value with the combination of FFN and CL and a high negative predictive value. For SPs the combination of both tests provides a better estimate of the risk of PTB within seven days and therefore could be integrated into the decision to hospitalize high risk women, use acute tocolysis and antenatal lung maturation or identify safely women at low risk and avoid overtreatment. 
For TPs the combination also seems to be superior, but in the sense that if either test is positive hospitalisation should occur. This underlines furthermore the high-risk situation for PTB in twin pregnancies, but should be interpreted with caution due to the small numbers of TP.

In contrast to many other countries cervical ultrasound assessment is mostly always available $24 \mathrm{~h}$ in Swiss obstetric departments. The combination of both tests might be more expensive than one test alone, but the higher specificity and negative predictive value allows reducing costs from unnecessary interventions.

Because the etiology of preterm labor is multifactorial, using multiple biomarkers from distinct biologic pathways will better predict the risk of preterm labor. Furthermore, combining non-invasive tools such as a physical or ultrasound finding may improve the ability of specific biomarker in predicting outcome [16].

Our findings concerning the properties of FFN and sonographic CL are in agreement with the previous reports considering the two tests in isolation [4] [8] [17] [18]. The results are in contrast to the study of Tsoi et al., where CL was the only significant contributor to the prediction of delivery within 7 days. FFN did not improve the prediction [12]. This prospective observational study, however, included women up to 36 weeks of gestation and $32 \%$ of the patients were of Afrocaribbean ethnicity. A limitation of our study is its retrospective nonrandomized design. In this context, a potential source of bias is represented by the fact that test results were available to clinicians and may have influenced clinical decision making. A possible indication in that direction is that patients with negative tests appear to have received tocolytics significantly less frequently. Although relatively small, our sample is one of the largest among published studies on this topic.

\section{Conclusion}

In singleton and twin pregnancies with PTL, both tests are significantly associated with a prediction of preterm birth within seven days. However, the combination of the FFN and sonographic CL in PTL outperforms either test. Our data suggest that a combination of FFN-testing and CL measurement is recommendable for singleton and twin pregnancies, but large prospective studies are needed too. There are a number of research projects and clinical trials currently underway in Europe evaluating the properties of these tests [19].

\section{References}

[1] Iams, J.D., Goldenberg, R.L., Meis, P.J., Mercer, B.M., Moawad, A., Das, A., et al. (1996) The Length of the Cervix and the Risk of Spontaneous Premature Delivery. National Institute of Child Health and Human Development Maternal Fetal Medicine Unit Network. New England Journal of Medicine, 334, 567-572.

http://dx.doi.org/10.1056/NEJM199602293340904

[2] Leitich, H., Egarter, C., Kaider, A., Hohlagschwandtner, M., Berghammer, P. and Husslein, P. (1999) Cervicovaginal Fetal Fibronectin as a Marker for Preterm Delivery: A Meta-Analysis. American Journal of Obstetrics \& Gynecology, 180, 1169-1176. http://dx.doi.org/10.1016/S0002-9378(99)70612-5

[3] Lockwood, C.J., Senyei, A.E., Dische, M.R., Casal, D., Shah, K.D., Thung, S.N., et al. (1991) Fetal Fibronectin in Cervical and Vaginal Secretions as a Predictor of Preterm Delivery. New England Journal of Medicine, 325, 669-674. http://dx.doi.org/10.1056/NEJM199109053251001

[4] Crane, J.M. and Hutchens, D. (2008) Transvaginal Sonographic Measurement of Cervical Length to Predict Preterm Birth in Asymptomatic Women at Increased Risk: A Systematic Review. Ultrasound in Obstetrics \& Gynecology, 31, 579-587. http://dx.doi.org/10.1002/uog.5323

[5] Honest, H., Forbes, C.A., Durée, K.H., Norman, G., Duffy, S.B., Tsourapas, A., et al. (2009) Screening to Prevent Spontaneous Preterm Birth: Systematic Reviews of Accuracy and Effectiveness Literature with Economic Modelling. Health Technology Assessment, 13. http://dx.doi.org/10.3310/hta13430

[6] Gomez, R., Romero, R., Medina, L., Nien, J.K., Chaiworapongsa, T., Carstens, M., et al. (2005) Cervicovaginal Fibronectin Improves the Prediction of Preterm Delivery Based on Sonographic Cervical Length in Patients with Preterm Uterine Contractions and Intact Membranes. American Journal of Obstetrics \& Gynecology, 192, 350-359. http://dx.doi.org/10.1016/j.ajog.2004.09.034

[7] van Baaren, G.J., Vis, J.Y., Wilms, F.F., Oudijk, M.A., Kwee, A. and Porath, M.M. (2014) Predictive Value of Cervical Length Measurement and Fibronectin Testing in Threatened Preterm Labor. Obstetrics \& Gynecology, 123, 11851192. http://dx.doi.org/10.1097/AOG.0000000000000229

[8] DeFranco, E.A., Lewis, D.F. and Odibo, A.O. (2013) Improving the Screening Accuracy for Preterm Labor: Is the Combination of Fetal Fibronectin and Cervical Length in Symptomatic Patients a Useful Predictor of Preterm Birth? A Systematic Review. American Journal of Obstetrics \& Gynecology, 208, 233.e1-233.e6. 
http://dx.doi.org/10.1016/j.ajog.2012.12.015

[9] van Baaren, G.J., Vis, J.Y., Grobman, W.A., Bossuyt, P.M., Opmeer, B.C. and Mol, B.W. (2013) Cost-Effectiveness Analysis of Cervical Length Measurement and Fibronectin Testing in Women with Threatened Preterm Labor. American Journal of Obstetrics \& Gynecology, 209, 436.e1-436.e8. http://dx.doi.org/10.1016/j.ajog.2013.06.029

[10] Berghella, V., Baxter, J.K. and Hendrix, N.W. (2013) Cervical Assessment by Ultrasound for Preventing Preterm Delivery. Cochrane Database of Systematic Reviews, 2013, Article ID: CD007235.

http://dx.doi.org/10.1002/14651858.CD007235.pub3

[11] Berghella, V., Hayes, E., Visintine, J. and Baxter, J.K. (2008) Fetal Fibronectin Testing for Reducing the Risk of Preterm Birth. Cochrane Database of Systematic Reviews, 2008, Article ID: CD006843. http://dx.doi.org/10.1002/14651858.cd006843.pub2

[12] Tsoi, E., Akmal, S., Geerts, L., Jeffery, B. and Nicolaides, K.H. (2006) Sonographic Measurement of Cervical Length and Fetal Fibronectin Testing in Threatened Preterm Labor.Ultrasound in Obstetrics \& Gynecology, 27, 368-372. http://dx.doi.org/10.1002/uog.2723

[13] Tsoi, E., Akmal, S., Rane, S., Otigbah, C. and Nicolaides, K.H. (2003) Ultrasound Assessment of Cervical Length in Threatened Preterm Labor. Ultrasound in Obstetrics \& Gynecology, 21, 552-555. http://dx.doi.org/10.1002/uog.131

[14] Sanchez-Ramos, L., Delke, I., Zamora, J. and Kaunitz, A.M. (2009) Fetal Fibronectin as a Short-Term Predictor of Preterm Birth in Symptomatic Patients: A Meta-Analysis. Obstetrics and Gynecology, 114, 631-640. http://dx.doi.org/10.1097/AOG.0b013e3181b47217

[15] Hoesli, I., Tercanli, S. and Holzgreve, W. (2003) Cervical Length Assessment by Ultrasound as a Predictor of Preterm Labour-Is There a Role for Routine Screening? British Journal of Obstetrics and Gynecology, 110, 61-65. http://dx.doi.org/10.1046/j.1471-0528.2003.00032.x

[16] Hanna, N. and Kiefer, D. (2012) A Translational View of Biomarkers in Preterm Labor. American Journal of Reproductive Immunology, 67, 268-272. http://dx.doi.org/10.1111/j.1600-0897.2012.01112.X

[17] Schmitz, T., Maillard, F., Bessard-Bacquaert, S., Kayem, G., Fulla, Y., Cabrol, D., et al. (2006) Selective Use of Fetal Fibronectin Detection after Cervical Length Measurement to Predict Spontaneous Preterm Delivery in Women with Preterm Labor. American Journal of Obstetrics \& Gynecology, 194, 138-143. http://dx.doi.org/10.1016/j.ajog.2005.05.074

[18] Singer, E., Pilpel, S., Bsat, F., Plevyak, M., Healy, A. and Markenson, G. (2007) Accuracy of Fetal Fibronectin to Predict Preterm Birth in Twin Gestations with Symptoms of Labor. Obstetrics and Gynecology, 109, 1083-1087. http://dx.doi.org/10.1097/01.AOG.0000261896.20175.3a

[19] Chandiramani, M., Di Renzo, G.C., Gottschalk, E., Helmer, H., Henrich, W., Hoesli, I., et al. (2011) Fetal Fibronectin as a Predictor of Spontaneous Preterm Birth: A European Perspective. The Journal of Maternal-Fetal and Neonatal Medicine, 24, 330-336. http://dx.doi.org/10.3109/14767058.2010.496879 